\title{
AN ALGORITHM FOR PARTITIONS
}

\author{
MELVYN B. NATHANSON
}

ABSTRACT. A class of algorithms is described to represent the positive integers as sums of elements in a prescribed sequence of integers, and results are obtained on the densities of the integers that can be represented by such partitions with a bounded number of summands.

Let $1=a_{1}<a_{2}<a_{3}<\ldots$ be a strictly increasing sequence of positive integers, and let $A=\left\{a_{i}\right\}$. Every positive integer can be written in the form

$$
n=a_{i_{1}}+a_{i_{2}}+\cdots+a_{i_{k}},
$$

where the summands $a_{i_{j}} \in A$ are chosen by the following "algorithm with $p$ choices": If $a_{i_{1}}, a_{i_{2}}^{j}, \ldots, a_{i_{k-1}}$ have been chosen, then $a_{i_{k}}$ must be one of the $p$ largest possible elements of $A$, i.e. if $a_{s-1} \leq n-\left(a_{i_{1}}+\ldots+\right.$ $\left.a_{i_{k-1}}\right)<a_{s}$, then $a_{i_{k}} \in\left\{a_{s-1}, a_{s-2}, \ldots, a_{s-p}\right\}$. The number of summands in the shortest permissible partition ( $*$ ) of $n$ is called the p-length of $n$, and denoted $L_{p}(n)$. Let $A_{p}^{h}$ be the set of all positive integers $n$ such that $L_{p}(n) \leq h$. Clearly, $A_{p}^{1}=A$ for all $p$, and $A_{p}^{h} \subset A_{p+1}^{b}$ and $A_{p}^{h} \subset A_{p}^{h+1}$ for all $p$ and $h$.

For example, let $A=\left\{i^{2}\right\}$ be the sequence of squares. Then the "algorithm with 2 choices" gives four permissible partitions of 12 , namely, $9+1+1+1=4+4+4=4+4+1+1+1+1=4+1+1+1+1+1+1+$ $1+1$, and so $L_{2}(12)=3$.

Katai [1] has studied the special case $p=1$ when the representation (*) is unique.

Let $S$ be any set of positive integers, and let $S(N)$ denote the number of $s \in S$ with $s \leq N$. The lower asymptotic density of $S$ is

$$
d(S)=\lim _{N \rightarrow \infty} \inf S(N) / N .
$$

The set $S$ has density zero if $\lim _{N \rightarrow \infty} S(N) / N=0$.

In this paper we study the densities of the sets $A_{p}^{b}$. If $A$ has density zero, then in Theorem 1 it is proved that all of the sets $A_{p}^{b}$ have density zero. For example, if $k \geq 2$ and $A$ is the sequence of $k$ th powers, then Waring asserted and Hilbert proved that every positive integer is the sum of a bounded number of $k$ th powers. But the sequence of $k$ th powers has density

Received by the editors July 5, 1974 . $10 \mathrm{~A} 30$.

AMS (MOS) subject classifications (1970). Primary 10J99, 10L99; Secondary

Key words and phrases. Partitions, representation of integers, density of sequences. 
zero, and so Theorem 1 shows that no algorithm with $p$ choices is strong enough to settle Waring's problem. In Theorem 2 a quantitative estimate is obtained for the size of $A_{p}^{h}$ when $A$ is a sequence distributed like the sequence of $k$ th powers.

If $A$ has positive lower asymptotic density, then $A_{p}^{h}$ can be large. For example, if $A=\{1,2, \ldots, m-1\} \cup\{i m\}_{i=1}^{\infty}$, then $A$ has density $1 / m$ and $A_{p}^{h}$ is the set of all positive integers for any $p \geq 1$ and $b \geq 2$. We prove in Theorem 3 that if $d(A)>0$, then $\lim _{h \rightarrow \infty} d\left(A_{p}^{h}\right)=1$ for all $p$.

If $f$ and $\phi$ are functions of $n$, then $f=O(\phi)$ means that there exists a constant $c$ such that $|f(n)|<c \phi(n)$ for all $n$.

Theorem 1. If $A=\left\{a_{i}\right\}$ has density zero, then $A_{p}^{h}$ has density zero for all $p$ and $h$.

Proof. Fix $p$. For $M \geq a_{p}$, choose $j$ so that $a_{p} \leq a_{j-1} \leq M<a_{j}$; that is, $j-1=A(M)$. Let $b \geq 2$. If $a_{j-1}<n \leq M$, then $n \in A_{p}^{b}$ if and only if $n-$ $a_{j-i} \in A_{p}^{h-1}$ for some $i=1,2, \ldots, p$. But $n-a_{j-i} \leq M-a_{j-i}$, and so the number of such $n$ with $n-a_{j-i} \in A_{p}^{b-1}$ is not more than $A_{p}^{h-1}\left(M-a_{j-i}\right)$. Therefore,

$$
A_{p}^{h}(M)-A_{p}^{b}\left(a_{j-1}\right) \leq \sum_{i=1}^{p} A_{p}^{h-1}\left(M-a_{j-i}\right) \leq p A_{p}^{h-1}\left(M-a_{j-p}\right) .
$$

Applying this inequality with $M=a_{j}-1$, we obtain

$$
\begin{aligned}
A_{p}^{h}\left(a_{j}\right)-A_{p}^{h}\left(a_{j-1}\right) & \\
& =1+A_{p}^{h}\left(a_{j}-1\right)-A_{p}^{h}\left(a_{j-1}\right) \leq 1+p A_{p}^{h-1}\left(a_{j}-a_{j-p}\right)
\end{aligned}
$$

Let $N \geq a_{p}$. Then

$$
A_{p}^{h}(N)=A_{p}^{h}(N)-A_{p}^{h}\left(a_{A(N)}\right)+\sum_{j=p+1}^{A(N)}\left\{A_{p}^{h}\left(a_{j}\right)-A_{p}^{h}\left(a_{j-1}\right)\right\}+A_{p}^{h}\left(a_{p}\right)
$$

$$
\leq p A_{p}^{h-1}\left(N-a_{A(N)-p+1}\right)+p \sum_{j=p+1}^{A(N)} A_{p}^{h-1}\left(a_{j}-a_{j-p}\right)+A(N)+A_{p}^{h}\left(a_{p}\right) .
$$

Let the sequence $A$ have density zero. Clearly, $A_{p}^{1}=A$, and so $A_{p}^{1}$ has density zero. Suppose that $A_{p}^{h-1}$ has density zero for some $b \geq 2$. Choose $\epsilon>0$. Then there exists a constant $M_{0}$ such that $A_{p}^{b-1}(M) \leq M_{\epsilon} / 2 p^{2}$ for all $M \geq M_{0}$. If $M<M_{0}$, then $A_{p}^{h-1}(M) \leq M<M_{0}$. Therefore, $A_{p}^{h-1}(M) \leq M_{0}+$ $M_{\epsilon} / 2 p^{2}$ for all $M$. Let $N \geq a_{p}$. By inequality (**), we have 


$$
\begin{aligned}
A_{p}^{h}(N) \leq & p A_{p}^{b-1}\left(N-a_{A(N)-p+1}\right)+p \sum_{j=p+1}^{A(N)} A_{p}^{h-1}\left(a_{j}-a_{j-p}\right)+A(N)+A_{p}^{h}\left(a_{p}\right) \\
\leq & \left.p M_{0}+\frac{\left(N-a_{A(N)-p+1}\right) \epsilon}{2 p^{2}}\right\} \\
& +p \sum_{j=p+1}^{A(N)}\left\{M_{0}+\frac{\left(a_{j}-a_{j-p}\right) \epsilon}{2 p^{2}}\right\}+A(N)+A_{p}^{h}\left(a_{p}\right) \\
\leq & p M_{0} A(N)+p\left\{N+\sum_{j=A(N)-p+2}^{A(N)} a_{j}\right\} \epsilon / 2 p^{2}+A_{p}^{h}\left(a_{p}\right)+A(N) \\
\leq & p M_{0} A(N)+N \epsilon / 2+A_{p}^{h}\left(a_{p}\right)+A(N) .
\end{aligned}
$$

Since $A$ has density zero, there exists $N_{0}>a_{p}$ such that $\left\{\left(p M_{0}+1\right) A(N)+\right.$ $\left.A_{p}^{h}\left(a_{p}\right)\right\}<N \epsilon / 2$ for all $N \geq N_{0}$. Then $A_{p}^{h}(N) / N<\epsilon$ for all $N \geq N_{0}$, and so $A_{p}^{h}$ has density zero. The theorem follows by induction on $h$.

Theorem 2. If $A=\left\{a_{i}\right\}$ satisfies:

(i) $A(N)=O\left(N^{1-\theta}\right)$ for $0<\theta<1$,

(ii) $a_{i}-a_{i-1}=O\left(i^{\mu}\right)$ for $\mu>1$, and

(iii) $(1+\mu)(1-\theta) \leq 1$,

then $A_{p}^{h}(N)=O\left(N^{1-\theta^{h}}\right)$, where the implied constant depends on $p$ and $h$.

Proof. Let $a_{0}=0$. Then $N \leq \Sigma_{i=1}^{A(N)+1}\left(a_{i}-a_{i-1}\right)$, and so, by (i) and (ii), we have $N \leq c N^{(1-\theta)(1+\mu)}$ for some constant $c$. It follows that $(1-\theta)(1+\mu) \geq$ 1 , and so, by (iii), we have $\mu=\theta /(1-\theta)$. Clearly, $A_{p}^{1}(N)=A(N)=O\left(N^{1-\theta}\right)$ and so the theorem holds for $h=1$. Suppose that $A_{p}^{h-1}(N)=O\left(N^{1-\theta^{h-1}}\right)$ for some $b \geq 2$. If $a_{i}-a_{i-1}=O\left(i^{\mu}\right)$, then $a_{i}-a_{i-p}=O\left(i^{\mu}\right)$, where the implied constant depends on $p$. By inequality $(* *)$ we have

$$
\begin{aligned}
A_{p}^{h}(N) & \leq p \sum_{j=p+1}^{A(N)+1} A_{p}^{h-1}\left(a_{j}-a_{j-p}\right)+A(N)+A_{p}^{h}\left(a_{p}\right) \\
& \leq p \sum_{j=p+1}^{A(N)+1} A_{p}^{h-1}\left(O\left(j^{\mu}\right)\right)+O\left(N^{1-\theta}\right) \\
& \leq \sum_{j=p+1}^{A(N)+1} O\left(j^{\mu\left(1-\theta^{h-1}\right)}\right)+O\left(N^{1-\theta}\right) \\
& \leq A(N) O\left(A(N)^{\mu(1-\theta b-1)}\right)+O\left(N^{1-\theta}\right) \\
& \leq O\left(N^{1-\theta}\right) O\left(N^{(1-\theta) \mu(1-\theta b-1)}\right)=O\left(N^{1-\theta^{h}}\right) .
\end{aligned}
$$

The theorem follows by induction on $h$. 
Note that if $A$ is the sequence of $k$ th powers, then $A(N)=O\left(N^{1 / k}\right)=$ $O\left(N^{1-(k-1) / k}\right)$ and $i^{k}-(i-1)^{k}=O\left(i^{k-1}\right)$.

Theorem 3. If $d(A)>0$, then $\lim _{h \rightarrow \infty} d\left(A_{p}^{h}\right)=1$ for all $p$.

Proof. Since $d(A)>0$ and $a_{1}=1$, there exists $\alpha>0$ such that $A(N) / N$ $>a$ for all $N$. In this case, Katai [1] has proved that for all $p$ and $N$,

$$
a \sum_{n=1}^{N} L_{p}(n) \leq N .
$$

But also

$$
\alpha h\left(N-A_{p}^{h}(N)\right) \leq \alpha \sum_{n=1}^{N} L_{p}(n) .
$$

Let $\epsilon>0$, and choose $h>1 /\left(\alpha_{\epsilon}\right)$. Then

$$
A_{p}^{h}(N) / N \geq 1-1 / \alpha h>1-\epsilon
$$

for all $N$. Then $d\left(A_{p}^{h}\right) \geq 1-\epsilon$ for all $h \geq 1 /\left(a_{\epsilon}\right)$, and so $\lim _{h \rightarrow \infty} d\left(A_{p}^{h}\right)=1$.

\section{REFERENCE}

1. I. Kátai, Some algorithms for the representation of natural numbers, Acta Sci. Math. (Szeged) 30 (1969), 99-105. MR 39\#1393.

SCHOOL OF MATHEMATICS, THE INSTITUTE FOR ADVANCED STUDY, PRINCETON, NEW JERSEY 08540

DEPARTMENT OF MATHEMATICS, SOUTHERN ILLINOIS UNIVERSITY, CARBONDALE, ILLINOIS 62901

Current address: Department of Mathematics, Brooklyn College (CUNY) Brooklyn, New York 11210 